2017-09-01

Non-linear modelling of a heaving point absorber: The surge effect

\author{
Miquel, AM
}

http://hdl.handle.net/10026.1/9933

10.1016/j.jjome.2017.07.002

International Journal of Marine Energy

All content in PEARL is protected by copyright law. Author manuscripts are made available in accordance with publisher policies. Please cite only the published version using the details provided on the item record or document. In the absence of an open licence (e.g. Creative Commons), permissions for further reuse of content should be sought from the publisher or author. 


\title{
Non-linear modelling of a heaving point absorber: the surge effect
}

\author{
Miquel A.M. ${ }^{\mathrm{a}, *}$, Antonini A. ${ }^{\mathrm{b}}$, Archetti R. ${ }^{\mathrm{a}}$, Bozzi S. ${ }^{\mathrm{c}}$, Lamberti A. $^{\mathrm{a}}$ \\ ${ }^{a}$ DICAM - University of Bologna - Viale del Risorgimento 2, Bologna (BO) 40122, \\ ITALY \\ ${ }^{b}$ Marine Institute - Plymouth University - Marine Building, Drake Circus, Plymouth, \\ Devon, PL4 8AA, UK \\ ${ }^{c}$ DEIB - Polytechnic Institute of Milan - Piazza Leonardo da Vinci 32, Milano (MI) \\ 40133, ITALY
}

\begin{abstract}
This paper presents a numerical model that simulates the behaviour of an offshore point absorber wave energy converter (WEC). The model receives $1^{\text {st }}$ order irregular waves as input and delivers instantaneous displacements, velocities and power as output. The model outputs are strongly non-linear due to the nature of some parts of the device, such as the power take off system (PTO), the mooring wires and the drag forces exerted on the wet bodies.

Two different devices are modelled, a two-body device consisting in a floating buoy attached to a linear generator placed at the sea bed and a three-body device, which also includes a submerged sphere located halfway from the float and the generator. For each device, the model takes into account either the heave mode only or the heave and surge modes combined.

The devices have been tuned to the Mediterranean wave climate, taking particular attention to the floater dimensions and to the geometrical design of the PTO, which has been redesigned to adapt to the newly introduced surge conditions.
\end{abstract}

\footnotetext{
*Corresponding author

Email address: adria.morenomiquel@unibo.it (Miquel A.M. )
} 
For the two-body device, although the dynamic behaviour changes when the surge is included, no relevant differences are observed regarding the power production. When studying the three-body device, results show two clear trends. For high waves, the surge leads to a decrease in the production, whereas for smaller waves it affects positively the power absorption. Overall, the negative contribution is more relevant but also less frequent, leading to no substantial change in the power production.

Including the surge mode in the model does not give significant variations in production rates and therefore, may be neglected only for energy production assessment. However, it should always be taken into account at the design stage.

Keywords: Wave Energy, Surge Effect, Non-linear Numerical Modelling, Mediterranean Sea, Wave Power Production, Point Absorber, Linear Generator

\section{Introduction}

Energy from the oceans is getting closer to become a reality in the renewable energy scenarios and not only where the energy resource is abundant (offshore the Atlantic coasts). New concepts keep appearing [1, 2] and at the same time some other WECs have reached the pre-commercial stages [3] showing that the research carried in this field is very broad, diverse and still open.

In the past decade a lot of effort has been put into device development and the research on the estimation of the wave energy potential has also grown, giving a more detailed picture of resource availability. Several studies have been published assessing wave energy along the oceans' coasts [4, 5] and more recently in milder seas [6], such as the Mediterranean and Black Sea $[7-11]$.

Nowadays, a number of full-scale wave energy devices have been deployed in real seas and several others are at the end of their development phase $[12,13]$. Most of them have been installed in moderate to high latitudes off the western coasts of Europe and America. Wave energy exploitation in less energetic climates can be achieved in several ways: by scaling existing WECs [14], by properly designing the power take off, as discussed in [15] or by specifically designing a novel device, as proposed in this paper. Currently, only very few attempts have been made to exploit wave energy in 
the Mediterranean Sea. A scaled prototype of an OWC has been installed in Reggio Calabria a few years ago and recently it has been announced that a prototype of an oscillating body is going to be deployed in the Tyrrenean Sea [16].

In the Mediterranean basin, estimations based on both, wave measurements and wave hind-casts, showed that the mean annual wave power ranges between 4 and $12 \mathrm{~kW} / \mathrm{m}$. The highest values occur in the south-western Aegean Sea, which is characterized by a relatively long fetch and strong winds. In Italy, two main wave climates can be identified: high waves coming mostly from the II and III quadrants on the western coast and smaller waves mainly coming from the north in the eastern coast. As a result, the annual average wave power is around $2 \mathrm{~kW} / \mathrm{m}$ off the Adriatic coast and between 3 and $5 \mathrm{~kW} / \mathrm{m}$ off the Tyrrhenian coast. The most energetic sites were identified in small offshore islands and in specific locations of Sicily and Sardinia, where the mean wave power reaches $10 \mathrm{~kW} / \mathrm{m}[9,14,17,18]$. Moreover, wave data analysis has shown that the wave climate in the Mediterranean Sea is characterized by high waves and high persistence of storms, but not by long-wave conditions.

A WEC specifically designed for the Tyrrhenian Sea should have the best performance for relatively short wave periods [18]. Some point absorber WECs with linear generators are currently being studied and developed in Europe and North America. Two promising technologies that already reached an advanced development stage are the Archimedes Wave Swing device, developed by the company AWS Ocean Energy (www.awsocean.com) and the Seabased wave energy converter, developed by the Swedish Centre for Renewable Electric Energy Conversion of the Uppsala University (www.seabased.com). The Seabased WEC consists of a buoy connected by a rope to a linear generator [19]. The vertical buoy's motion brought about by the ocean waves is transferred to the piston and the stator coils react to the piston's moyement inducing alternate current. The springs connecting the bottom of the translator to the foundation act as a restoring force, thus behaving as an energy storage unit. Each single device has a relatively low power output and therefore, the idea is to install several devices in arrays of many units.

In this study, a two-body and a three-body device have been modelled. They both share the same bottom anchored PTO, characterized by a permanentmagnet generator with a highly non-linear behaviour, where the PTO's translator is one of the modelled bodies. Both devices have also another body, a 
cylindrical floater $\mathrm{n}$ The third body is add inertia to the ods without increas connected to the of

Figure 1: Device's Layout.

The PTO of the studied WEC is inspired on the Seabased's linear generator [19, 21-24]; more specifically, it is simulated using the model presented in [21] and adopted by [20] afterwards. The hydrodynamic behaviour of different types of floaters has been investigated in [25], where two different buoy geometries: hemisphere-cylindrical and cone-cylindrical with 18 different internal configurations have been analysed.

A study on the optimal buoy dimensions is presented in [20]. Two cylindrical buoys with different diameters and drafts are compared to select the best buoy size for several representative locations in the Italian Seas. The power output is then maximised by adding a submerged body connected to the floating buoy, which allows the shifting of the natural frequency of the system in order to match it with the typical wave frequency of the studysites. Furthermore, this body is placed at a depth where it can barely feel the presence of waves. Thus, the energy loss caused by radiation is negligible. Only one degree of freedom was modelled (Heave) for the whole device and 


\begin{tabular}{c|ccc} 
& N. of Bodies & N. of DoFs & Surge \\
\hline A & 2 & 2 & X \\
B & 2 & 3 & $\checkmark$ \\
C & 3 & 3 & X \\
D & 3 & 5 & $\checkmark$
\end{tabular}

Table 1: Studied WEC devices.

regular waves were used to simulate the sea state conditions. A further study on the optimization of the numerical modelling if the device was presented in $[26]$.

The aim of this paper is to present a comprehensive analysis and discussion of the modelling of the considered WEC under irregular wave sea states. For the first time the surge effect is modelled and quantified by estimating the energy production when considering a point absorber WEC excited in the horizontal direction and comparing it to the simplified model, which takes into account only the heave mode. See [20, 24].

The comparison of the generic performance of the devices is presented depending on the number of bodies (floater + piston or floater + piston + submerged sphere) and degrees of freedom (heave only or heave + surge). Table 1 summarizes all the combinations studied in this work.

The presented comparison yields a large number of combinations, therefore the computational effort of the model has been a relevant issue in this study. According to the available computational resources (i.e:server), a reasonable computational cost has been reached by parallelizing the code and through implementing the Prony's approach.

The paper is organized as follows: in section 2, the mathematical model is presented, in subsection 2.1 the theoretical approach is described, in subsection 2.2 the theoretical basis are applied according to the requirements of the analysed devices, highlighting the introduced novelties. In section 3, a recapitulation of the application sites characterisation is shown. Subsequently, section 4 goes through the optimization process of the device. Afterwards, the obtained results are presented in section 5, giving a general overview of the devices performance in subsection 5.1 and the site application cases in subsection 5.2. Finally, in the last section, some discussions and conclusions are drawn, focusing on the differences between the improved variants compared to the simplified ones, on the energy production and on the device 
performance.

\section{Modelling}

\subsection{Theory}

The dynamic behaviour of the wave energy converter is expressed through the general governing equation of motion (1), which links the components from different nature altogether.

$$
m \ddot{z}(t)=F_{e}(t)+F_{r}(t)+F_{h}(t)+F_{\text {moor }}(t)+F_{\text {drag }}(t)+F_{p t o}(t)
$$

where $m$ is the mass of the system, $z$ refers to the coordinate system of the model, $F_{e}(t)$ is the wave excitation force, $F_{r}(t)$ is the radiation force, $F_{h}(t)$ is the hydrostatic restoring force, $F_{\text {moor }}(t)$ is the force exerted by the mooring system, $F_{\text {drag }}(t)$ is the viscous drag force and $F_{p t o}(t)$ is the resistant force due to the power take off action. The excitation force is obtained by convoluting the impulse response function $f_{e}(t)$ and the sea surface elevation $\eta(t)$ as stated in equation (2):

$$
F_{e}(t)=f_{e}(t) * \eta(t)
$$

The term expressing the resistance of the body due to the radiated waves is composed by two terms, a convolution between the body velocity and its impulse response function and an inertial term, as shown in equation (3):

$$
F_{r}(t)=-m_{\infty} \ddot{z}(t)-k(t) * \dot{z}(t)
$$

where $m_{\infty}$ is the added mass at infinite frequency, the body velocity $\ddot{z}(t)$ and $k(t)$, which is the radiation impulse response function that acts as kernel of the convolution. According to the Kramers-Kronig relations, takes the form shown in eq. (4), as deeply discussed in Falnes, p.31-36, [27].

$$
k(t)=\frac{2}{\pi} \int_{0}^{\infty} \mathrm{B}(\omega) \cos (\omega \mathrm{t}) \mathrm{d} \omega
$$

where $\omega$ is the monochromatic wave frequency and $B(\omega)$ is its radiation damping coefficient. The hydrostatic force $F_{h}(t)$ acting on a cylindrical shape can be linearised on the heave mode as follows, when its centre of gravity is coincident with the origin of the coordinate system $z(t)$ : 


$$
F_{h}(t)=-\rho g A_{w} z(t)
$$

where $\rho$ is the seawater density, $g$ is the gravity acceleration and $A_{w}$ is the water plane area of the cylinder. Since the PTO is fixed on the seabed, the mooring forces are expressed as the non-linear elastic forces occurring at the lines, which interconnect the different bodies of the device.Therefore, they have been modelled as stiff springs acting only when in tension.

$$
F_{\text {moor }}(t)= \begin{cases}-K_{\text {line }} \Delta l(t) & \text { for } \Delta l(t)>0 \\ 0 & \text { Otherwise }\end{cases}
$$

where $K_{\text {line }}$ is the elastic constant of the wire and $\Delta l(t)$ is the relative displacement between bodies. The drag forces have been described according to the Morison expression for oscillatory flows:

$$
F_{\text {drag }}(t)=-\frac{1}{2} \rho C_{d} A_{d}|\dot{V}(t)-\dot{z}(t)|(\dot{V}(t)-\dot{z}(t))
$$

where $C_{d}$ is the drag coefficient, which depends on the shape of the body and has been chosen according to the tabulated values in [28], assuming a value of 1.1 for the cylinder and 0.47 for the sphere. $A_{d}$ is the area of the body projected perpendicularly to the flow direction and $\dot{V}(t)$ is the fluid velocity.

The Power Take Off system introduces three different forces, two mechanical ones and an electromagnetic one. The PTO has a spring attached to the bottom that stores part of the energy and helps to smooth the translator's displacements. To enhance its survivability, the generator includes two endstop mechanism, consisting of an upper and a lower spring, in order to avoid any damage when the device be subjected to stormy conditions. The electromagnetic resistant force is derived from the instantaneous electric power, which in turn is yielded from the electric currents and tensions found in the electric equivalent circuit of the stator coils. Equations (8), (9) and (10) describe forces mentioned above:

$$
\begin{gathered}
F_{\text {spring }}(t)=-K_{\text {pto }} z(t) \\
F_{\text {end }}(t)= \begin{cases}-K_{\text {end }}\left(z(t)-Z_{\text {lim }}\right) & \text { for }|z(t)|>\left|Z_{\text {lim }}\right| \\
0 & \text { Otherwise }\end{cases}
\end{gathered}
$$




$$
F_{M}(t)=\frac{\sum_{i=1}^{3} U_{i}(t) I_{i}(t)}{\dot{z}(t) \mu}
$$

where $K_{p t o}$ is the elastic constant of the spring attached to the translator, $K_{\text {end }}$ is the elastic constant of the end-stop spring, and $Z_{\text {lim }}$ is the activation coordinate of the end-stop. $U_{i}(t)$ and $I_{i}(t)$ are the electric tension and current of the $i^{t h}$ phase of the equivalent circuit respectively, which have been obtained applying the Faraday's laws. The electric field is found using the analytical model presented by [21], which uses the Maxwell's equations that describe the electromagnetic induction phenomenon in the stator-translator structure. The total instantaneous electric power is the sum of the power for any electric phase, each of them computed as the product of the tension times the current. By dividing the power by the translator velocity $\dot{z}(t)$ and the overall generator's efficiency $\mu$, the electromagnetic resistant force is yielded. As already applied in [20].

\subsection{Model}

All the mathematical expressions presented in the previous section are written in the generic form and they have been adapted to each device and model variant as exposed on the introduction chapter. Some specific modifications need to be done too in order to meet the numerical requirements.

Each DoF of the system is expressed mathematically by an equation of motion. Hence, the total number of degrees of freedom per device determines the dimension of the matrix system of the model, varying from a two-dimension system for the simplest case ( 2 bodies, 2 DoFs ) up to a fivedimension system (3 bodies, 5 DoFs). In order to give better understanding, the left side of equation (1) is displayed below for the most complete situation i.e. three-body device considering the heave and surge modes. Each body is specified with the superscript 1, 2 and 3 for the buoy, submerged sphere and translator respectively. The surge mode is specified with the subscript 1 , and the heave mode with the subscript 3.

$$
\left(\begin{array}{ccccc}
m_{\infty 11}^{1}+m^{1} & m_{\infty 13}^{1} & 0 & 0 & 0 \\
m_{\infty 31}^{1} & m_{\infty 33}^{1}+m^{1} & 0 & 0 & 0 \\
0 & 0 & m_{\infty 11}^{2}+m^{2} & m_{\infty 13}^{2} & 0 \\
0 & 0 & m_{\infty 31}^{2} & m_{\infty 33}^{3}+m^{2} & 0 \\
0 & 0 & 0 & 0 & m^{3}
\end{array}\right)\left(\begin{array}{c}
\ddot{z}_{1}^{1}(t) \\
\ddot{z}_{3}^{1}(t) \\
\ddot{z}_{1}^{2}(t) \\
\ddot{z}_{3}^{2}(t) \\
\ddot{z}_{3}^{3}(t)
\end{array}\right)=\ldots
$$


where $m_{\infty}$ 's is the added mass at infinite frequency and $\ddot{z}(t)$ 's is the body acceleration. The hydrodynamic coefficients $F e(\omega), B(\omega)$ and $A(\omega)$ (the excitation force coefficient, the radiation damping coefficient and the added mass coefficient respectively), which are frequency dependent, have been obtained using the open source BEM method software called NEMOH (http://lheea.ec-nantes.fr/doku.php/emo/nemoh/start?\&\#nemoh). As seen on the previous section, these coefficients are used to compute the impulse response functions, which in turn work as kernels for convolution as seen in equation (4).

The matrix system of equations of motion is a set of differential equations, which have been integrated over time in order to obtain the displacements and velocities of the system. The commercial software Matlab ${ }^{\circledR}$ has been used, applying a fourth order ODE solver based on Runge-Kutta's method. Some practical problems arise when using this approach, the main drawback is the high computational cost of the simulation. This is mainly due to the internal convolutions of the wave excitation force and radiation effect, which have to be pre-calculated at each time step. Indeed, this fact forces the algorithm to run in a fixed time step, rising even more the computational cost.

By means of the Prony's approach the computational time has been approximately halved. This method avoids the use of the convolutions by adding $N$ virtual DoFs $\left(I_{i}(t)\right)$ to the system, where $i=1 \ldots N$ and then assuming that the summation of all these new DoFs approximates the product of the avoided convoltuion, as in eq. (12) shows:

$$
F_{\text {rad }}=\sum_{i=1}^{N} I_{i}(t)
$$

Despite the increase of the size of the system, which in turn implies a growth of the computational time, the benefits are by far larger than the drawbacks. More insight on the Prony's method can be found at [29-31]

The viscous drag of the device has been modelled for all degrees of freedom, with some particularities. The vertical drag component corresponding to the heave mode of the cylinder is negligible according to [32], since the relative velocity of the body with respect to the fluid is very small. The velocity of the fluid around submerged body has been considered equal to zero since the sphere is placed at a sufficient depth where the disturbance of the wave field is of insignificant relevance, according to [20]. 


\section{Description of the study sites}

The presented device has been tuned for wave conditions typical of closed seas, characterized by short waves and intense storms. In order to estimate the Annual Energy Production (hereinafter AEP) two specific sites off the Italian coasts have been selected, where wave data are available and where the wave energy converter is supposed to be deployed. The selected sites, Alghero and Mazara del Vallo are located, respectively, on the West side of Sardinia's and Sicily's coasts. The wave potential in Alghero is $9.1 \mathrm{~kW} / \mathrm{m}$ and $4.7 \mathrm{~kW} / \mathrm{m}$ in Mazara del Vallo, [9]).

The characterization of the wave climate and the wave energy potential in terms of sea states is presented in [20]. Original data is provided by the Italian Buoy Network (http://www.idromare.it), operatively collecting wave data since 1989. Wave climate data shows that the prevalent sea states are characterized by relative small waves: in Alghero and Mazara $H_{S}$ is below 1 $\mathrm{m}$ during approximately $60 \%$ of the year. The peak periods with the highest probability of occurrence are around $6 \mathrm{~s}$, confirming that short waves prevails in the Mediterranean climate, as the results of [20].

The model takes irregular waves as input. The spectrum that best represents the current sea states is the JONSWAP with a $\gamma$ parameter set equal to 2, as shown in [18]. Furthermore, in order to account for the spectral energy associated with the frequencies lying outside the simulation range, an algorithm applying energy compensation has been used. It is based on the ratio of the theoretical $m_{0}$ related to the theoretical spectrum $(S(\omega))$ and the value $m_{0}^{*}$ that comes from the numerical integration of the truncated JONSWAP spectrum, $\left(S^{*}(\omega)\right)$. The aim is to generate a modified truncated JONSWAP spectrum $\left(\left(S^{+}(\omega)\right)\right)$ which has the same total energy $\left(m_{0}^{+}\right)$as the analytic one. Equations 13 - 16 describe the approach, while in Fig. 2 an example for a JONSWAP spectrum $\left(H_{S}=5 \mathrm{~m}-T_{P}=10 \mathrm{~s}-\gamma=2\right)$ is presented.

$$
\begin{gathered}
m_{0}=\int_{0}^{\infty} S(\omega) d \omega \\
m_{0}^{*}=\int_{0}^{f_{u}} S^{*}(\omega) d \omega \quad \text { where } \quad f_{u}=3.3 \frac{2 \pi}{T_{p}} \\
S^{+}(\omega)=S(\omega) \frac{m_{0}}{m_{0}^{*}}
\end{gathered}
$$




$$
m_{0}^{+}=m_{0}=\int_{0}^{f_{u}} S^{+}(\omega) d \omega
$$

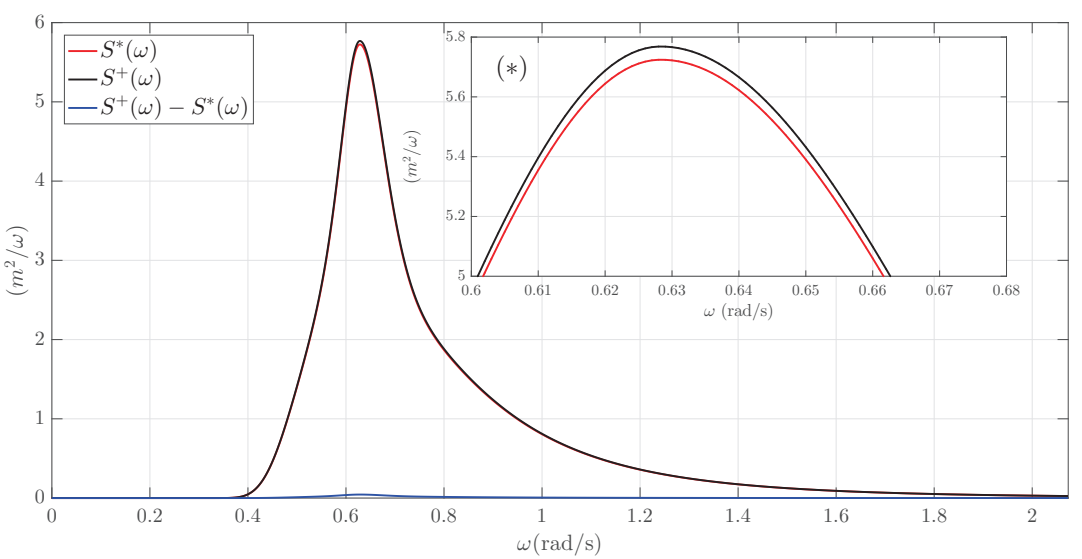

Figure 2: Comparison between the theoretical and the numerically integrated JONSWAP spectra. (*) Zoom at the spectra's peaks

Furthermore, in order to perform an effective analysis of the device dimensioning a new indicator has been used; the climatic spectrum. It is computed as the weighted average of each JONSWAP spectrum that characterises the wave climate matrix at the selected locations; adopting the frequency of occurrence as the weighting parameter. Equations 17 and 18 expose the procedure followed to compute the climatic spectrum.

$$
\begin{gathered}
S_{C}(\omega)=\sum_{j=1}^{P} \sum_{i=1}^{N} f_{i j}^{o c} S_{i j}(\omega) \\
f_{i j}^{o c}=\frac{O C_{i j}}{\sum_{j=1}^{P} \sum_{i=1}^{N} O C_{i j}}
\end{gathered}
$$

Where $S_{i j}(\omega)$ is the JONSWAP spectrum with $\gamma=2$ given an $H_{S}$ class 280 indexed $\mathrm{i}$ and $T_{P}$ class indexed $\mathrm{j}, f_{i j}^{o c}$ is the frequency of occurrence of the 281 aforementioned spectrum and $O C_{i j}$ is the actual occurrence in hours. The 
data used to compute the wave climates have been obtained from the measurements given by the RON (Rete Ondametrica Nazionale) [33]. The climatic spectrum aggregates two different time scales, giving a good insight on which are the most energetic frequencies at both sites globally and thus, is used to tune the device performance.

\section{Dimensioning \& Tuning}

In first approach, the device has been modelled only in heave and with the PTO translator built-in with the floater. Influence on the floater's shape and draft has been analysed using three different geometries. A cylinder and two composed geometries, a cylinder with a conical base and a cylinder with spherical base. The optimal configuration has been found to be the regular cylinder with $\varnothing=5 \mathrm{~m}$ and draft of $d=2.75 \mathrm{~m}$, as described in [25].

\subsection{Free oscillation tests}

The aim of the submerged body is to maximize the power output by shifting the natural period of the system towards the prevailing wave periods of the study sites sea states. The shape of the chosen submerged body is a sphere. After the selection of the shape, the last characteristic to be determined is the radius. For floating bodies, standard procedure to identify the natural modes of the system is the free oscillating test. This, consists in varying the initial position from the equilibrium state and observe the evolution over time under total absence of external disturbances; in this case, represented by a flat sea. The length of the test has been set to $100 \mathrm{~s}$, after this time it has been observed that the oscillations are completely damped and the system has reached back the equilibrium state. Setting the equilibrium condition at the point $(0,0)$ of the coordinate system $\left(z_{1}^{1}(t), z_{3}^{1}(t)\right)$, the initial displacement of the buoy has been established at $(-1.25,-1.25)$, hence for both, surge and heave.

Figure 3 shows the results of the free oscillations test for four different variants of the device, the first one without sphere, and the rest accounting with a sphere of different diameter. Figure 3.a) shows the evolution of the system over time while Fig. 3.b) shows the result of the frequency analysis. Furthermore, in black, the climatic spectra $S_{C}(\omega)$ of the deployment sites are shown. 

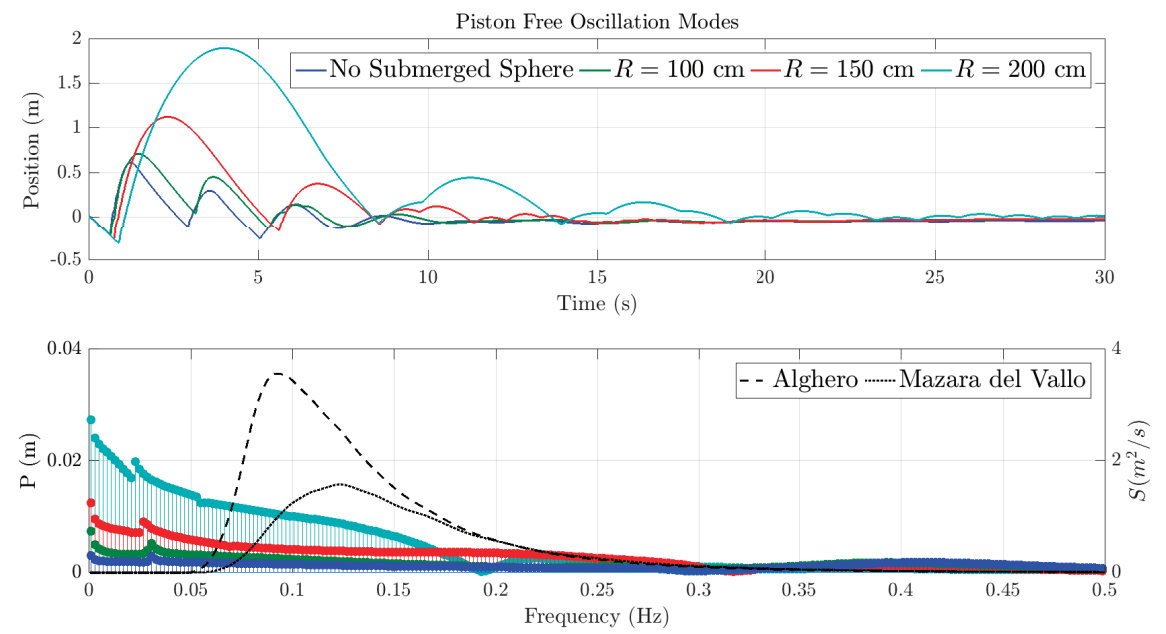

Figure 3: Free Oscillation test of the PTO's translator. a) Influence of the sphere vs. time. b) Spectral analysis of oscillations and climatic spectra from Alghero and Mazara del Vallo

As expected, a strong non-linear behaviour is observed in Fig. 3.a) and no clear resonance is detected in Fig. 3.b). Nonetheless, the influence of the sphere is clear on the dynamic response of the system. Oscillations in the piston increase, in period and amplitude, as the radius of the sphere grows. Judging from the area of interest (the frequency range of the climate spectra) the optimal solution appears to be the device accounting with a 2.00 $m$ radius sphere since it shows the highest amplitudes. However, not only the oscillations grow with the radius but so the non-linearities do, giving place to several undesired effects such as, slamming due to the wires, endstop mechanism activation, translator oscillating outside the productive area. Therefore, the configuration with the sphere of radius $1.50 \mathrm{~m}$ delivers the best performance and assures smooth operation conditions of the device since its response is stable throughout the whole range of interested frequencies.

\subsection{PTO's Design}

The surge motion directly causes a variation on the oscillatory regime of the piston. An extra horizontal component is introduced at the buoy and that makes the absolute displacements larger. The absolute displacement of the buoy is then transferred to the piston through the steel wire. This causes 
a shift of the piston mean oscillatory position (see Fig. 3.a) and causes a decrease of the energy production as the PTO is designed to oscillate around zero. In order to solve this undesired effect the PTO has been redesigned geometrically.

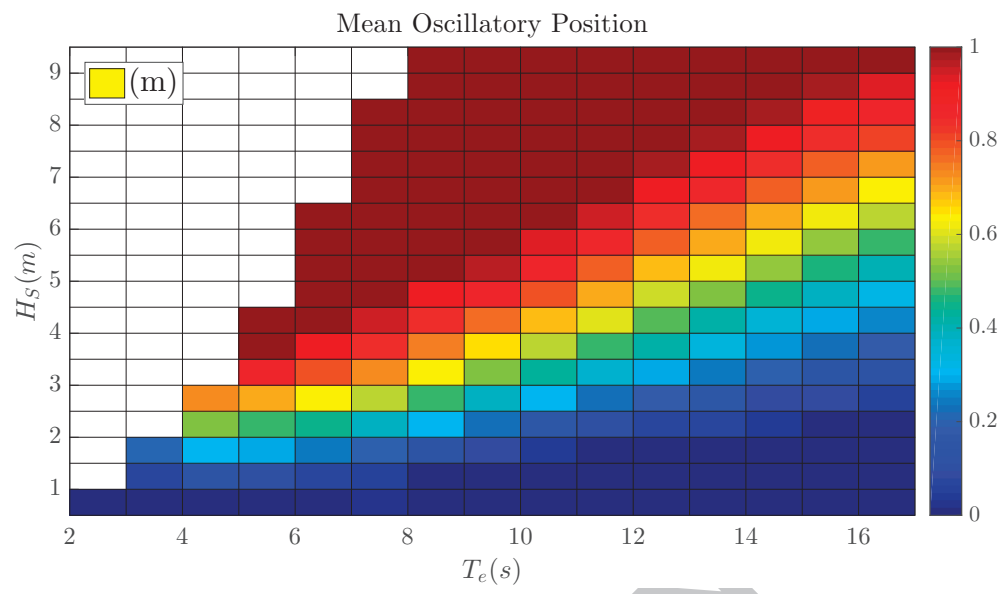

Figure 4: Piston's Average Position at each Sea State.

Figure 4 shows the mean oscillatory position of the piston for each simulated condition; at the typical working conditions $\left(T_{P}=[5.5-7.5](s) \&\right.$ $\left.H_{S}=[1.0-2.5](m)\right)$, the average oscillatory position is about $\bar{x}=0.25 \mathrm{~m}$. According to such preliminary result, the piston is extended by $2 \bar{x}$ and the upper part of the stator is also lengthened by $\bar{x}$. This combination allows the lower bound of the maximal production rate to remain untouched, whereas the upper bound of the minimal production rate is extended by $2 \bar{x}$. Furthermore, the upper end stop position is also shifted by $\bar{x}$ to ensure that the piston smooth motion conditions are not affected by this change. 
Figure 5: PTO layout. a) Original form. b) Translator modification. c) Translator\&Stator modification.

346 The active production area is the surface of the stator, entirely or par347 tially, containing the translator. If divided by the total area of the stator, the 348 Active Area Ratio (AAR) is obtained. Figure 6 maps the differences in the 349 active production area of the PTO for the original and the modified PTO. 350 Figure 5 shows the differences between the original design of the PTO and 351 the optimized one. 


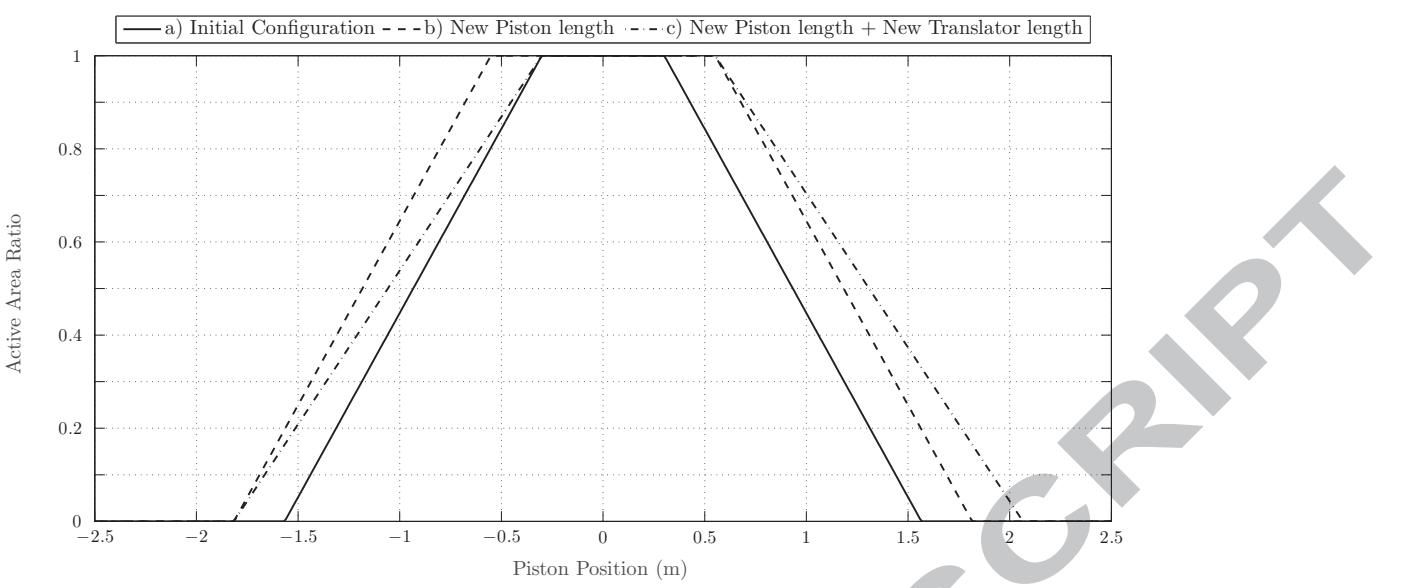

Figure 6: PTO Active Production Area Ratio vs. Piston Displacement .

To sum up, the modifications applied to the PTO regard only the geometrical configuration, keeping the electromagnetic properties unvaried, as described in [21]. Table 2 summarizes the geometrical and electromagnetic properties of the linear generator.

PTO Parameters

\begin{tabular}{c|c}
\hline Nominal Power $(\mathrm{kW})$ & 10 \\
Nominal Speed $(\mathrm{m} / \mathrm{s})$ & 0.67 \\
Translator length $(\mathrm{m})$ & 2.367 \\
Stator length $(\mathrm{m})$ & 1.514 \\
Translator mass $(\mathrm{kg})$ & 1000 \\
Width of stator sides $(\mathrm{m})$ & 0.4 \\
Number of sides $(-)$ & 4 \\
Pole width $(\mathrm{mm})$ & 50 \\
Tooth width $(\mathrm{mm})$ & 8 \\
Magnetic Field in tooth $(\mathrm{T})$ & 1.55 \\
Generator Resistance $(\Omega)$ & 0.3735 \\
Generator Inductance $(\mathrm{mH})$ & 11.5 \\
DC Voltage $(\mathrm{V})$ & 200 \\
Efficiency $\mu(-)$ & 0.791 \\
\hline
\end{tabular}

Table 2: Electric generator properties [20]. 


\subsection{Duration of the Simulations}

In order to achieve a reliable estimate of the power absorption a standard length of the simulations needs to be defined. Due to the wide range of simulated sea states, a fixed duration of the simulations is not appropriate, as the system may reach the device production power stabilization at different times depending on the input wave characteristics. A suitable indicator of the length of the simulations was found to be the number of waves. It was determined that after 1000 waves a constant value of mean power production was reached for each of the simulated sea states. Taking into account the high level of uncertainty at this stage of the research, the authors believe that an error in power output estimation below $5 \%$ can be considered acceptable. Hence, the duration of the simulations was set equal to the number of waves necessary to obtain a value of power output differing by less than $5 \%$ from the value obtained with 1000 waves. Fig. 7 shows the deviation from the 1000-wave value versus the number of simulated waves, for the system D (see Table 1). It can be noticed that the desired level of accuracy is reached for a number of waves equal to 350. The same results were found for the other WEC systems, so the duration of the simulations was set equal to 350 waves for all the studied devices.

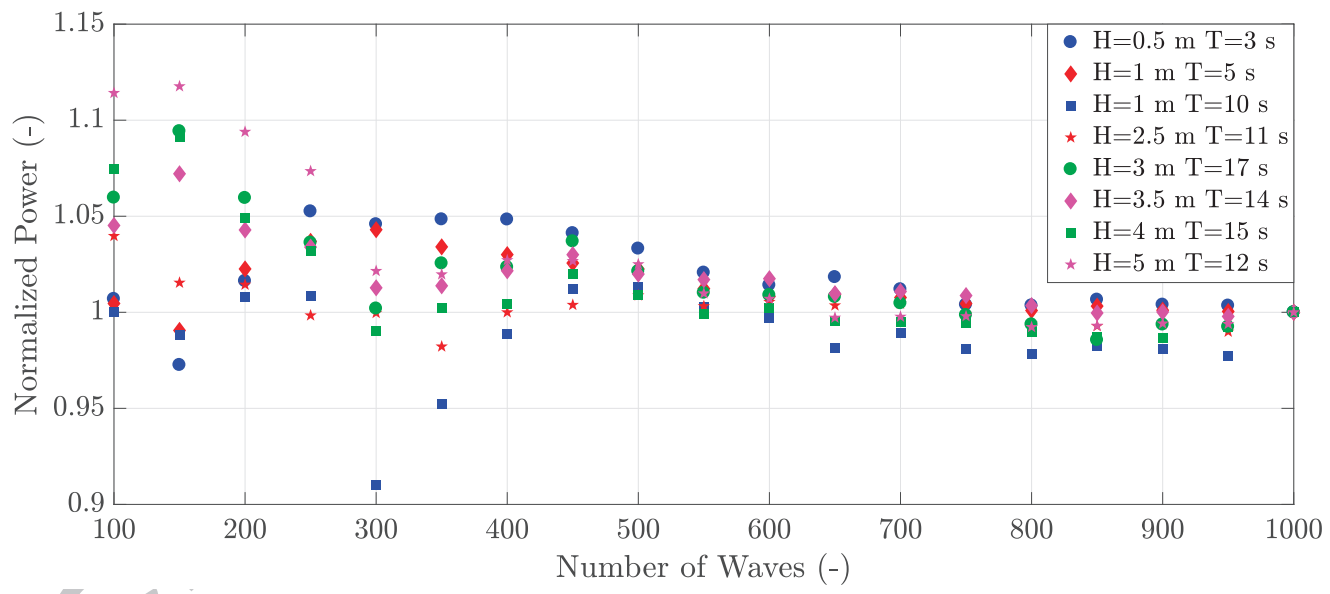

Figure 7: Power output deviation from the 1000-wave value vs Number of Waves 


\section{Results}

\subsection{Generic}

102 simulations, corresponding to the full range of sea states that characterize the selected locations wave climate, have been simulated for each of the different device variants, see tab. 1. For each simulation the following parameters are extracted: displacement and velocity time-series of each device part and instantaneous power. The production of the device is obtained by averaging the instantaneous power over the time-series, for any specific sea state. When combining all the output powers for each different sea state, characterised by the peak period $\left(T_{P}\right)$ and the significant wave height $\left(H_{S}\right)$, a two-dimensional matrix is obtained, which is commonly named power matrix. In order to assess the device general performance, the power matrix of each variant is shown in Fig. 8.

A)

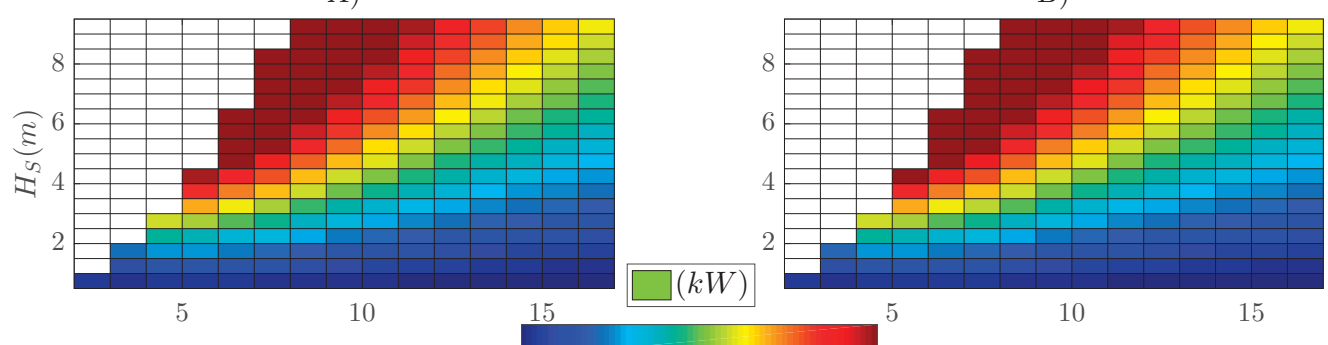

C) 0

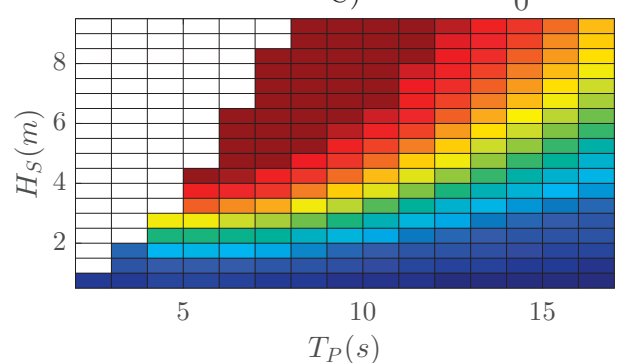

5

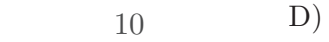

D)

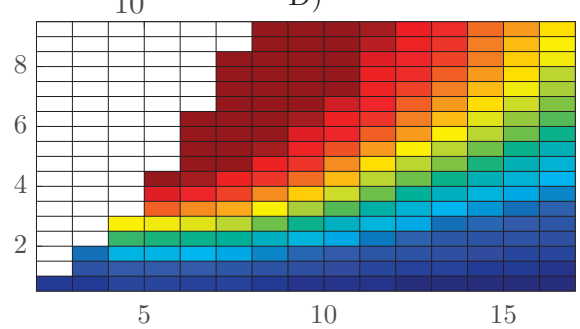

$T_{P}(s)$

Figure 8: Power matrices for each variant of the device. A) Two bodies only heave, $B)$ two bodies heave \& surge, $C$ ) three bodies only heave and D) three bodies heave \& surge. (as in table 1)

Figure 8.A displays the two-body variant free to move only in heave (A in tab. 1), Fig. 8.B presents the two-body variant accounting for the heave 
and surge modes ( $\mathrm{B}$ in tab. 1), Fig. 8.C summarizes the performance of the three-boy device only in heave $(\mathrm{C}$ in tab. 1) and Fig. 8.D reveals the behaviour of the most complete model, accounting for three bodies and five degrees of freedom ( $\mathrm{D}$ in tab. 1). All the power matrices show the expected behaviour. The general trend shows higher production rates at higher and steeper waves; furthermore, an increase of the produced power is noticed when the third body is added. Yet, no evident differences are observed when the surge is introduced. Therefore, a more thorough analysis is needed to study such effects in depth. In order to quantify the influence that the submerged sphere has in the power production, variants $\mathrm{A} \& \mathrm{C}$, and D \& B are confronted by subtracting their power output for every sea state. Such results are shown in Fig. 9.

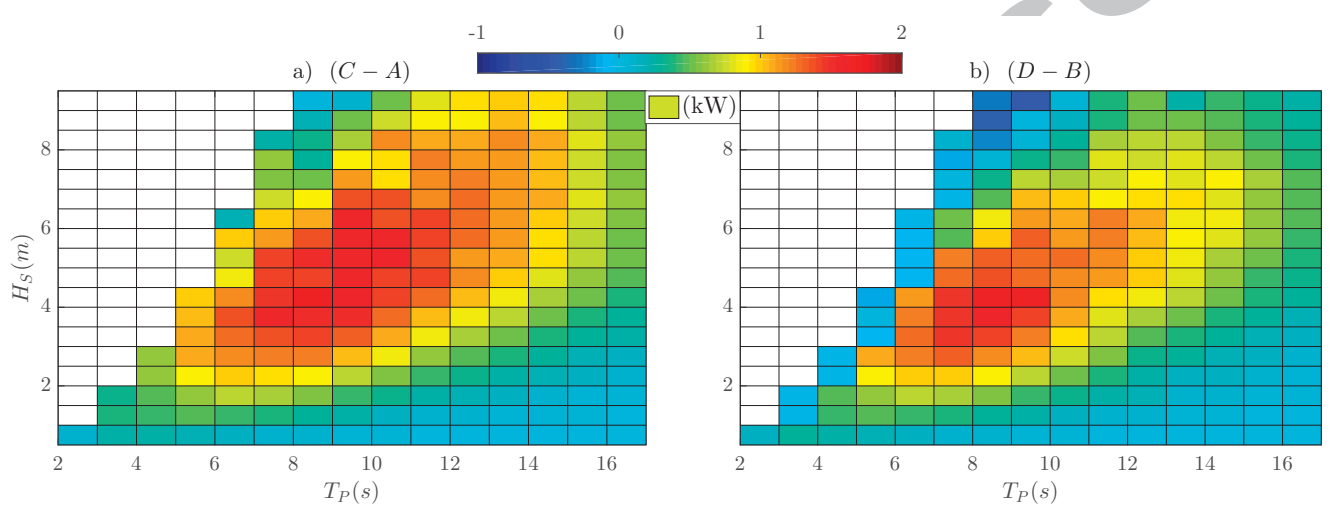

Figure 9: Power matrix difference between the two \& three-body device. a) Heave only. b) Heave \& surge.

A clear patch is observed in Fig. 9.a), having a production peak between $T_{P}$ 's 7 and 9 seconds, shifting the most productive area towards higher periods, just as predicted in the previous chapter. The same trend is identified in Fig. 9.b) even though the surge effect seems to mitigate it substantially. In addition, for very steep waves, this trend is even reversed and the surge effect is revealed to be counter productive because of the negative values of the production rate. This means that the addition of the submerged sphere is not always optimal, specifically if the device is to be deployed in a location where wind seas are predominant.

To better explore the device response, the same methodology as in the previous figure has been applied, subtracting A - B and C - D. By doing 
so, the pure surge effect can be analysed for both, the two and three-body device. See Fig. 10.

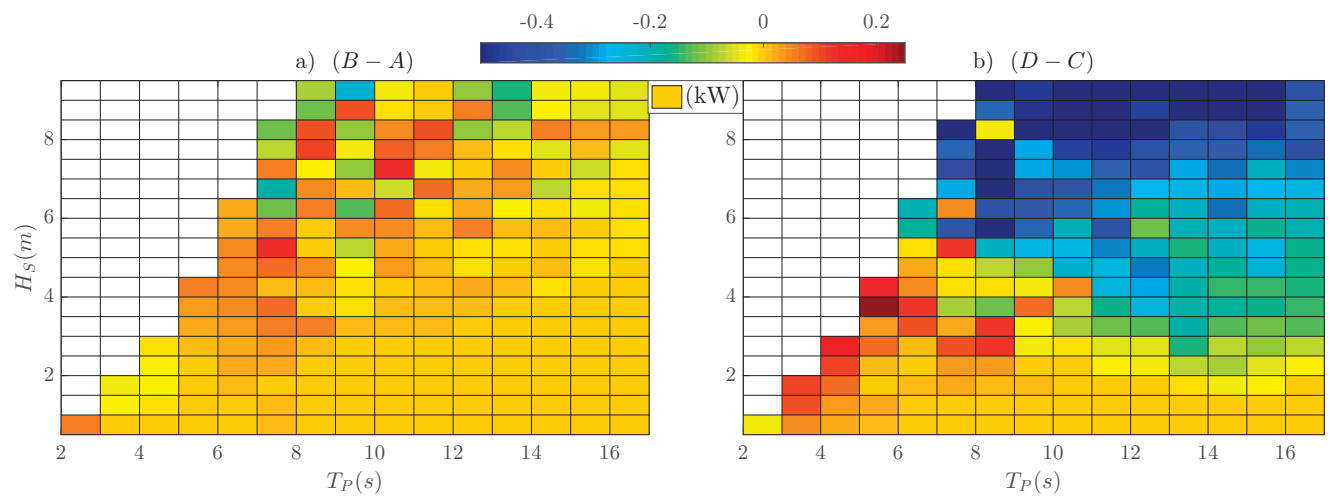

Figure 10: Power matrix difference between the heave-only mode and the heave \& surge mode. a) Two-body device. b) Three-body device.

Figure 10 shows a different behaviour between devices when the surge mode is modelled. The three-body device shows a clear positive trend in production rates for small steep waves, typically $H_{S}<4.5 \mathrm{~m}$ and $T_{P}<7 \mathrm{~s}$. On the contrary, a decrease of the production is detected for flatter waves and getting more intense for $H_{S}>4.5 \mathrm{~m}$ (Fig. 10.b ). The two-body device shows no predominant trend, having the strongest variations in the steep-wave region. Hence, both devices appear to have high sensitivity to wave steepness since in both figures the most extreme values are found at the steep wave area and the minimum variation is obtained at the flat-wave area. Consequently, the difference in each device's power production has been studied according to wave steepness. See Fig. 11. 


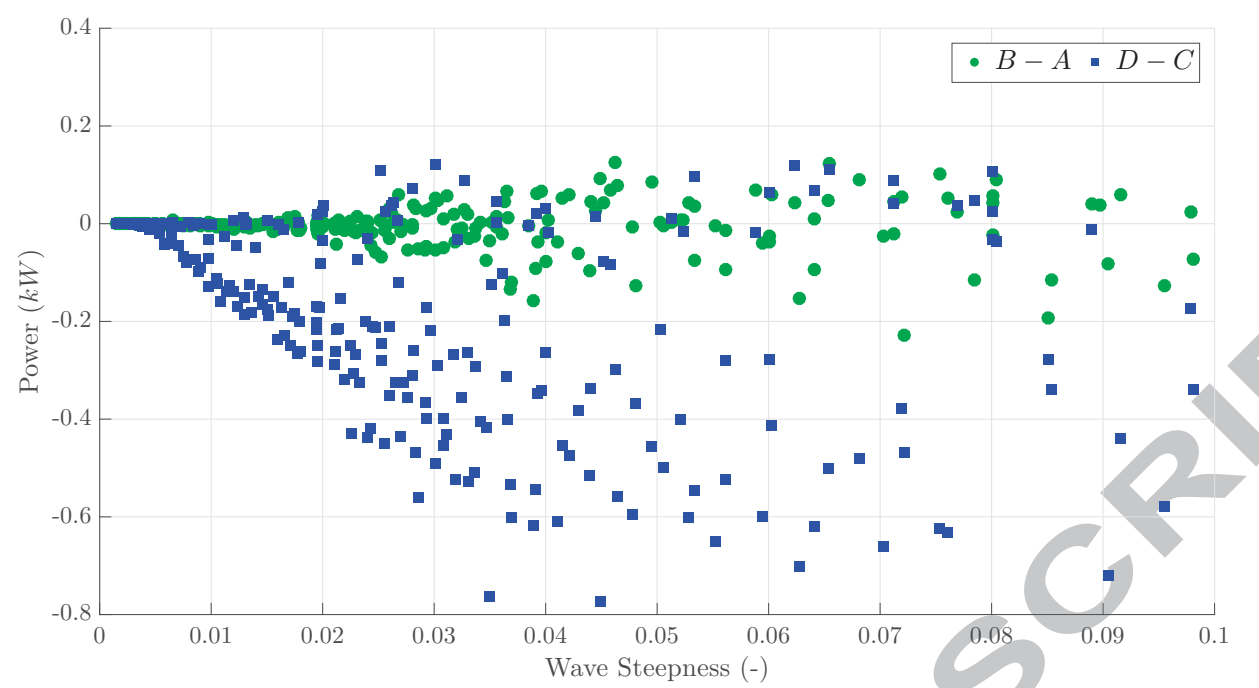

Figure 11: Power output difference vs. wave steepness for each device, green circle and blue square for two-body and three-body device respectively.

The two-body device reveals very low surge-related sensitivity to wave steepness, since the scatter cloud mean is approximately null and its dispersion is rather low. On the contrary, the blue-dotted cloud has a clear wave steepness akin trend, which confirms that the inclusion of the sphere has an evident negative contribution when modelling the surge mode, as the power difference increases with the wave steepness.

Although the buoy is the body in direct contact with waves, the electric production is carried out by the PTO's piston. This, in the case of the threebody device, is greatly influenced by the submerged sphere. To study this behaviour, an analysis of three parameters concerning the piston's dynamics has been carried out. The aforementioned parameters are the following: the active area ratio of the PTO (described in the previous section), the average velocity of the piston and average amplitude of the piston's oscillations. The AAR gives very good insight, not only for the amplitude of the oscillations but also for the offset of the centre of such oscillations with respect to the equilibrium position. Furthermore, the average oscillation amplitude helps to complete the analysis on this regard, since a joint study of both parameters allows to obtain a detailed picture on the piston regime. Finally, it is important to consider as well the average piston's velocity since it is directly linked to the power output through the magnetic induction laws. 
Figures 12 and 13 have been computed following the same procedure as in Fig. 10. The values shown are $\operatorname{AAR}(B)-A A R(A)$ and $A A R(D)-A A R(C)$, for the a) section of Figs. 12 and 13 respectively. The average piston run at the b) section and the average piston velocity for section c).
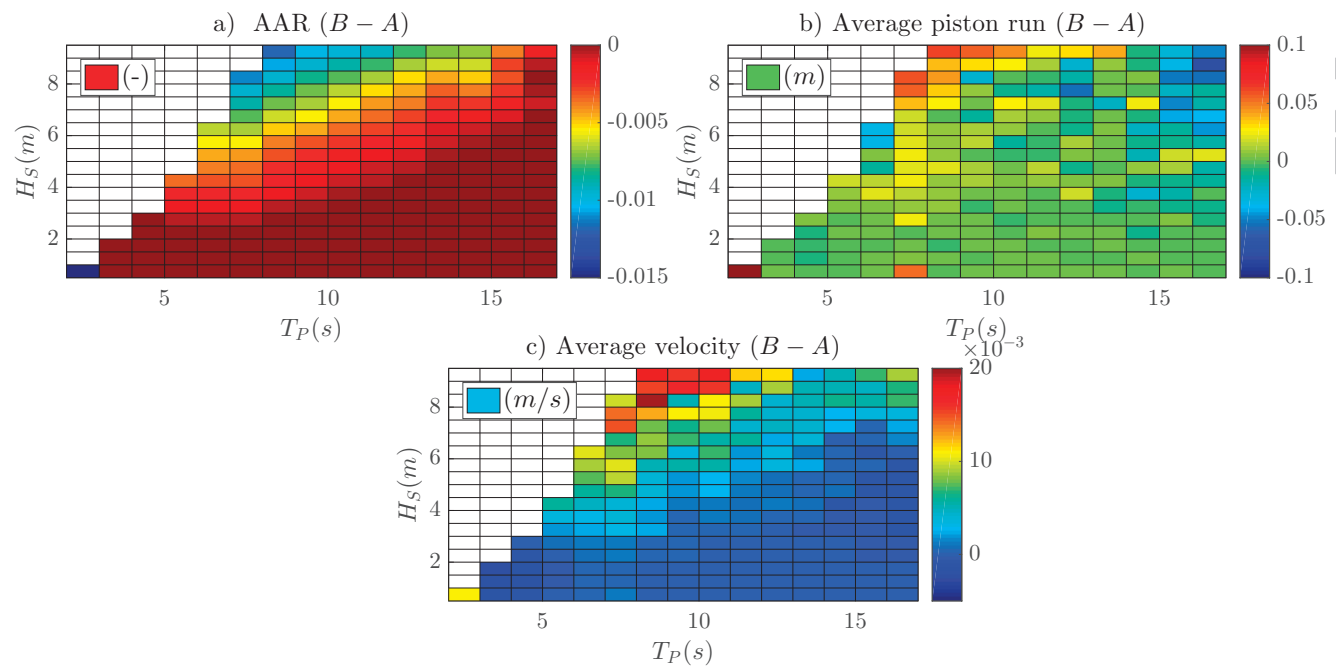

Figure 12: Two-body device difference matrices between heave-only mode and heave \& surge mode. a) Active Area Ratio, b) Average amplitude of the piston's motion, c) Average piston's velocity

The combination of positive average piston run and negative AAR differences given at the top-left corner of the matrix (high and steep waves) means that the piston presents larger oscillations when the surge is taken into account but, it is doing so outside the range where electricity is effectively produced. However, the velocity differences are also positive at the same area, meaning a higher electricity production. Considering the values, it can be realized that differences are actually very small. This, brings about high uncertainty upon the dominance of a specific parameter over the other. As a matter of fact, this was already observed in Figs. 10.a) and 11, where no clear conclusion can be drawn whether the surge effect is either positive or negative for the two-body device. 

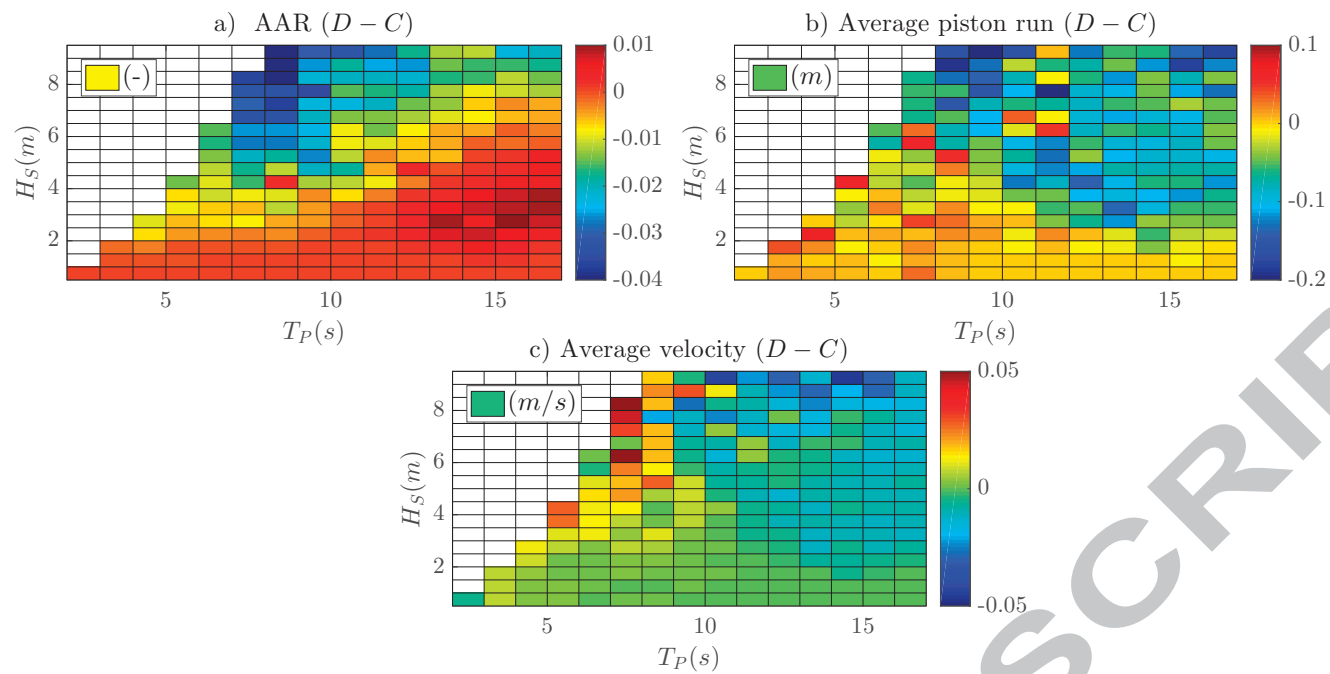

Figure 13: Three-body device difference matrices between heave-only mode and heave \& surge mode. a) Active Area Ratio, b) Average amplitude of the piston's motion, c) Average piston's velocity

For the three-body device instead, a clear pattern can be distinguished for steep waves. The AAR difference is negative for steep waves, which implies that the piston oscillates less effectively when the surge mode is taken into account. Nevertheless, the velocity differences are positive, which means it oscillates slightly faster. The piston run is negative for high waves, indicating a more frequent activation of the end-stop mechanism, leaving no doubt to the negative influence of the surge at that region of the matrix, as already confirmed by Figs. 10.b) and 11. For the other regions of the matrix no substantial difference is encountered other than a slight increase of the piston run and AAR for smaller waves.

\subsection{Site-Specific}

The average energy production of the simulated devices has been computed for the two selected sites, Alghero and Mazara del Vallo. A 20 year long data record provided by the RON [33] has been used to compute the climate matrices of the deployment sites. These, are then crossed with the power matrix of the device to obtain the average energy production. Results are shown in table 3. 


\begin{tabular}{ccc|cc} 
Variant & N. of Bodies & Surge & Alghero & Mazara del Vallo \\
\hline A & 2 & X & 12.89 & 9.34 \\
B & 2 & $\checkmark$ & 12.91 & 9.35 \\
C & 3 & X & 17.00 & 12.28 \\
D & 3 & $\checkmark$ & 17.04 & 12.38
\end{tabular}

Table 3: Annual Energy Production for the four variants (tab. 1) at the selected locations. All units are $M W h / y$.

The three-body device has a higher electricity production, the increase is about 30\% for Alghero and 32\% for Mazara del Vallo, stating the shift in the resonance frequency induced by the submerged sphere towards more common sea state conditions. The surge has no influence on the long-term electricity production, results are almost identical either for Alghero or Mazara del Vallo, denoting that the major differences in power production identified in section 5.1 occur for rather improbable sea states at these locations. The differences in annual energy production depending on the number of bodies will affect the resulting cost of the electricity of the two technical solutions, as shown by [34].

\section{Conclusions}

With the aim to estimate the feasibility of wave energy conversion in the Mediterranean Sea this paper thoroughly analyses the body dynamics, with particular focus on the surge effect and in the energy production of a point absorber WEC. The model runs in the time domain, uses irregular waves, is able to handle multi-body systems with various degrees of freedom and delivers the instantaneous electric power, which is later used to obtain both, generic and site-specific performance indicators. In order to increase its computational efficiency, the code has been parallelized and the prony's method has been adopted, reducing the total simulation-time by an order of magnitude.

A sensitivity analysis on the dimension of the submerged body has been performed by running several free oscillation tests. These, have confirmed that the optimal submerged body size is $R=1.50 \mathrm{~m}$. Figures 3 and 9 show that the resonant frequency of the system is shifted towards the most persistent sea state period range. On the one hand, the presence of the sub- 
merged body increases the electric production, which goes up to approximately 30\% when tanking into account both, heave only and heave and surge modes. On the other hand, it could lead to undesired effects from the technical/operational point of view, such as the increase of the working time of the end-stop survival mechanism or the slamming effects occurring in the interconnecting lines due to its large inertia. For all the stated above, it is reasonable to worry about the technical/economical feasibility of a point absorber with a submerged body disposed in such configuration, particularly when considering the surge, as no increase of the electricity production is found.

Slamming phenomena occurring in extreme wave events have been considered in the mathematical modelling by means of the end-stop mechanism and the steel wire modelling, see section 2.1. Nevertheless, their effect has not been deeply analysed due to their negligible contribution on mean annual energy production. Slamming effects have a major relevance in the reliability and survivability of the devices and hence should be adequately taken into account in WEC design. A relevant work including slamming restraint constraints in WEC modelling has been recently published by [35].

Another remarkable conclusion that can be drawn from the previous section is that considering the surge shows no relevant contribution to the electricity production, as presented in table 3 . Therefore, the surge mode may be neglected at early stages of development when modelling numerically the behaviour of a heaving point absorber for pure energy production assessment purposes. This, allows to use a simpler and more computationally efficient model that brings in more flexibility from the research point of view.

The shift of the piston not only affects the electric production directly, but also some technical aspects. Since the piston offset makes it easier to reach the limit position for survival of the device. Hence, for the same wave conditions, when considering the surge, the end-stop mechanism is activated sooner; and consequently, the electric production utterly decreases. Furthermore, the more the end-stop mechanism is working the higher the probability of breakdowns (slamming effects and high tensions in wires and springs). Consequently, it is reasonable to think that a shorter lifespan of the device and higher maintenance tasks mean higher costs in general. A possible way to reduce such an undesired effect and capsize this trend would involve the implementation control strategies, for instance, a moving stator which adapts to the mean oscillatory position of the translator.

However, it is crucial to acquire deep knowledge on how all the effects 
introduced by the surge influence the device.

The average annual production, is rather low for the single device. Therefore, the exploitation concept for this kind of the devices lies in the wave energy farm. Deploying a substantially elevated number of devices in arrays. Some studies $[36,37]$ conclude that, if well spatially distributed, a wave energy farm can produce at a higher rate than the single device. Since the available wave power resource in the Mediterranean Sea is much lower than in other areas of the planet, nowadays the only way to make wave energy exploitation feasible with point absorbers is by means of multiple-device plants.

\section{Acknowledgments}

We would like to acknowledge Professor Giuseppe Passoni for the very useful discussion.

\section{Bibliography}

[1] S. R. e Silva, R. P. F. Gomes, A. F. O. Falcão, Hydrodynamic optimization of the ugen: Wave energy converter with u-shaped interior oscillating water column, International Journal of Marine Energy 15 (2016) $112-126$.

[2] M. Dragić, M. Hofman, V. Tomin, D. Volk, V. Miškov, Model tests of sigma wave energy converter: The lessons learned, International Journal of Marine Energy 13 (2016) 96 - 129.

[3] M. Göteman, J. Engström, M. Eriksson, J. Isberg, Optimizing wave energy parks with over 1000 interacting point-absorbers using an approximate analytical method, International Journal of Marine Energy 10 (2015) $113-126$.

[4] J. Scruggs, P. Jacob, Harvesting ocean wave energy (science (1176)), Science 324 (5931) (2009) 1142.

[5] L. Rusu, C. G. Soares, Wave energy assessments in the Azores islands, Renewable Energy 45 (2012) 183-196. 
[6] A. Mirzaei, F. Tangang, L. Juneng, Wave energy potential assessment in the central and southern regions of the South China Sea, Renewable Energy 80 (2015) 454-470.

[7] F. Barbariol, A. Benetazzo, S. Carniel, M. Sclavo, Improving the assessment of wave energy resources by means of coupled wave-ocean numerical modeling, Renewable Energy 60 (2013) 462-471.

[8] F. Arena, V. Laface, G. Malara, A. Romolo, A. Viviano, V. Fiamma, G. Sannino, A. Carillo, Wave climate analysis for the design of wave energy harvesters in the Mediterranean Sea, Renewable Energy 77 (2015) $125-141$.

[9] L. Liberti, A. Carillo, G. Sannino, Wave energy resource assessment in the Mediterranean, the Italian perspective, Renewable Energy 50 (2013) 938-949.

[10] H. Keskin Citiroglu, A. Okur, An approach to wave energy converters in eregli on the wester black sea coast of turkey, Applied Energy 135 (2014) 738-747.

[11] A. Akpinar, M. Íhsan Kömürkü, Assessment of the wave energy resource of the black sea based on 15-year numerical hindcast data, Applied Energy 101 (2013) 502-512.

[12] A. Clément, P. McCullen, A. Falcão, A. Fiorentino, F. Gardner, K. Hammarlund, G. Lemonis, T. Lewis, K. Nielsen, S. Petroncini, M. T. Pontes, P. Schild, B. O. Sjöström, H. C. Sø rensen, T. Thorpe, Wave energy in Europe: current status and perspectives, Renewable and Sustainable Energy Reviews 6 (5) (2002) 405-431.

[13] A. F. D. O. Falcão, Wave energy utilization: A review of the technologies, Renewable and Sustainable Energy Reviews 14 (3) (2010) 899-918.

[14] S. Bozzi, R. Archetti, G. Passoni, Wave electricity production in Italian offshore: A preliminary investigation, Renewable Energy 62 (2014) 407416.

5] R. Harne, M. Schoemaker, B. Dussault, K. Wang, Wave heave energy conversion using modular multistability, Applied Energy 130 (2014) 148156. 
[16] G. Bracco, E. Giorcelli, G. Tedeschi, M. Mattiazzo, M. Molinas, Control Strategies for the ISWEC Wave Energy System, in: Ninth European Wave and Tidal Energy Connference (EWTEC), 2011.

[17] D. Vicinanza, P. Contestabile, V. Ferrante, Wave energy potential in the north-west of Sardinia (Italy), Renewable Energy 50 (2013) 506-521.

[18] R. Archetti, S. Bozzi, G. Passoni, Feasibility study of a wave energy farm in the western mediterranean sea: Comparison among different technologies, in: Proceedings of the International Conference on Offshore Mechanics and Arctic Engineering - OMAE, Vol. 5, 2011, pp. $447-452$.

[19] M. Leijon, O. Danielsson, M. Eriksson, K. Thorburn, H. Bernhoff, J. Isberg, J. Sundberg, I. Ivanova, E. Sjöstedt, O. A gren, K. E. Karlsson, a. Wolfbrandt, An electrical approach to wave energy conversion, Renewable Energy 31 (9) (2006) 1309-1319.

[20] S. Bozzi, A. M. Miquel, A. Antonini, G. Passoni, R. Archetti, Modeling of a point absorber for energy conversion in Italian seas, Energies 6 (6) (2013) 3033-3051.

[21] K. Thorburn, M. Leijon, Farm size comparison with analytical model of linear generator wave energy converters, Ocean Engineering 34 (5-6) (2007) 908-916.

[22] R. Waters, M. Rahm, M. Eriksson, O. Svensson, E. Stromstedt, C. Bostrom, J. Sundberg, M. Leijon, Ocean wave energy absorption in response to wave period and amplitude offshore experiments on a wave energy converter, IET Renewable Power Generation 5 (6) (2011) 465.

[23] M. Eriksson, R. Waters, O. Svensson, J. Isberg, M. Leijon, Wave power absorption: Experiments in open sea and simulation, Journal of Applied Physics 102 (8) (2007) 1-6.

[24] J. Engström, M. Eriksson, J. Isberg, M. Leijon, Wave energy converter with enhanced amplitude response at frequencies coinciding with Swedish west coast sea states by use of a supplementary submerged body, Journal of Applied Physics 106 (6). 
[25] A. Antonini, A. M. Miquel, R. Archetti, S. Bozzi, G. Passoni, Preliminary design of a point absorber with linear generator designed for energy production off the Italian coasts, in: Tenth European Wave and Tidal Energy Connference (EWTEC), 2013.

[26] A. M. Miquel, A. Antonini, R. Archetti, S. Bozzi, G. Passoni, Assessment of the surge effects in a heaving point absorber in the Mediterranean Sea, in: ASME 2014 33rd International Conference on Ocean, Offshore and Arctic Engineering, OMAE2014, June 8-13, San Francisco, California, USA, 2014, pp. 1-8.

[27] J. Falnes, Ocean waves and Oscillating systems, Vol. 30, Cambridge University Press, 2004.

[28] D. F. Y. B. R. Munson, T. H. Okiishi, Fundamentals of Fluid Mechanics, John Wiley \& Sons, Inc., 2006.

[29] J. F. Hauer, C. J. Demeure, L. L. Scharf, Initial results in Prony analysis of power system response signals, IEEE Transactions on Power Systems 5 (1) (1990) 80-89.

[30] J. F. Hauer, Application of Prony analysis to the determination of modal content and equivalent models for measured power system response, IEEE Transactions on Power Systems 6 (3) (1991) 1062-1068.

[31] F. J. Diemer, A PRONY ALGORITHM FOR SHALLOW WATER, Master's thesis, Massachusetts Institute of Technology (1987).

[32] L. Yang, J. Hals, T. Moan, Analysis of dynamic effects relevant for the wear damage in hydraulic machines for wave energy conversion, Ocean Engineering 37 (13) (2010) 1089 - 1102.

[33] [link].

URL http://www . telemisura.net

[34] V. Piscopo, L. Benassai, R. Della Morte, A. Scamardella, Towards a new cost-based design of heaving point absorbers., Internation Journal of Marine Energy (18) (2017) 15-29.

[35] V. Piscopo, G. Benassai, L. Cozzolino, R. Della Morte, A. Scamardella, A new optimization procedure of heaving point absorber hydrodynamic performance, Ocean Engineering (116) (2016) 242-259. 
[36] A. Babaritl, On the park effect in arrays of oscillating wave energy converters, Renewable Energy 58 (2013) 68 - 78.

[37] B. Child, V. Venugopal, Optimal configurations of wave energy device arrays, Ocean Engineering 37 (16) (2010) 1402 - 1417. 


\section{Highlights}

- Point absorber WEC modeled with several degrees of freedom per body.

- A 2-body and a 3-body device modeled in, irregular waves, heave and surge.

- Coupling of mechanic (hydrodynamic and lines) and electromagnetic forces (PTO).

- Comparison between only heaving device and heaving and surging device.

- Comparison and analysis of the production between the different configurations.

- Production assessment in the Mediterranean Sea, Sardinia and Sicily. 